\title{
Prise en compte de l'efficience humaine pour pallier les limites du Lean
}

\section{Considering human efficiency to overcome the limits of Lean}

\author{
Patrick Badets ${ }^{1}$, Christophe Merlo $^{2}$, Véronique Pilniere ${ }^{1}$ \\ ${ }^{1}$ Univ. Bordeaux, ESTIA Institute of Technology, 64210 Bidart, France, [p.badets ; v.pilniere]@estia.fr \\ ${ }^{2}$ Univ. Bordeaux, ESTIA Institute of Technology, IMS UMR 5218, 64210 Bidart, France, c.merlo@estia.fr
}

RÉSUMÉ. Le Lean est une démarche qui vise à éliminer des gaspillages au sein des activités de production. Plébiscitée par les entreprises, cette démarche permet d'obtenir des gains importants sur le court terme. Cependant, à moyen et long terme, ces résultats présentent des limites. Nos travaux de recherche visent à pallier ces limites. Pour cela, nous nous intéressons au modèle de performance adopté par les " décideurs " des entreprises Lean ainsi qu'aux représentations mentales qui sous-tendent ce modèle. Dans cet article, nous appuyons notre recherche sur une enquête auprès d'entreprises pratiquant la démarche Lean et ayant rencontré les limites évoquées, afin de comprendre ces limites. Nous proposons alors un nouveau modèle de performance Lean qui vise à pallier ces limites. Ce modèle est construit sur la vision que les opérateurs de production contribuent à la performance de l'entreprise par la création et le déploiement de modes opératoires pertinents au cours de leur activité de travail en contexte Lean. Nous proposons ensuite un cadre de modélisation qui permette de représenter les impacts de ce nouveau modèle de performance Lean sur la prise de décision d'un système de production Lean.

ABSTRACT. Lean is an approach that aims to eliminate waste in production activities. Recognized by companies, this approach yields significant gains in the short term. However, at medium and long term, these results have limitations. Our research work is focused on overcoming these limitations. So we studied the performance model adopted by the managers of Lean companies and the mental representations that underlie this model. In this paper, we support our research on a case study based on interviews with companies applying Lean approach and having met the mentioned limits. We then propose a new Lean performance model that attempts to overcome such limitations. This model is based on the vision that production operators contribute to the performance of the company through the creation and deployment of relevant operating modes during their work in Lean context. We also propose a modelling framework that allows to represent the impact of this new Lean performance model on the decision making in a Lean production system.

MOTS-CLÉS. Démarche Lean, modèle de performance, modélisation d'entreprise, GRAI.

KEYWORDS. Lean approach, performance model, enterprise modelling, GRAI.

\section{Introduction}

Les démarches de rationalisation se suivent au fil des décennies dans les entreprises qui les adoptent. Citons par exemple la démarche TPM (Total Productive Management), ISO 9001 ou le BPR (Business Process Reengineering). Il en est une qui, même ancienne, se démarque par l'ampleur de sa diffusion et l'attention qu'elle suscite toujours auprès des entreprises françaises : la démarche Lean. Les résultats sont cependant contrastés d'une entreprise à l'autre. Nos travaux visent à comprendre les facteurs de cause et contribuer à pallier certaines limites identifiées du Lean . Dans cet article nous allons dans un premier temps décrire le contexte de nos travaux et préciser la problématique que nous adressons, en rappelant brièvement ce qu'est la démarche Lean. Dans la section 3 nous étudierons le modèle de performance qui sous-tend la mise en œuvre du Lean. Ce modèle s'appuie à la fois sur une enquête menée auprès de dix entreprises pratiquant le Lean ainsi que d'un état de l'art pluridisciplinaire. En section 4 nous proposerons un nouveau modèle de performance, puis en section 5 un cadre de modélisation permettant de le mettre en œuvre pour la conduite d'une organisation de production, à travers un cas d'étude (section 6). 


\section{Contexte et problématique}

\subsection{Une démarche d'élimination des gaspillages qui attire les entreprises}

La démarche Lean trouve ses origines dans le Toyota Production System (TPS). Le TPS renvoie à des principes et des outils d'organisation de la production déployés par l'entreprise Toyota dans les années 1950. Dans cette période d'après-guerre, Toyota cherchait à concurrencer les entreprises américaines en adoptant une stratégique d'élimination des gaspillages au sein des activités de production [HOL 07]. Les gaspillages ciblés sont, par exemple, la réduction des stocks de production, la rationalisation des gestes des opérateurs ou bien la réduction des flux de matière considérés comme excessifs [OHN 88]. Ces gaspillages ont donné naissance à plusieurs principes et outils du Lean, comme par exemple le «juste à temps » qui consiste à fabriquer la quantité exacte demandée par le client au bon moment, ou bien le VSM (Value Stream Mapping) qui permet d'identifier plusieurs types de gaspillage, comme par exemple les stocks ou les temps d'attente par la schématisation des processus de production.

Jusque dans les années 1970, la stratégie de Toyota, axée sur la réduction des gaspillages, intéressait peu les entreprises occidentales [BOU 93]. En effet, la demande dépassait l'offre : la stratégie de l'économie d'échelle était privilégiée par rapport à une stratégie axée sur l'élimination des gaspillages. Or, dans les années 1970-1980, le contexte change : l'offre dépasse la demande [DUP 98]. La stratégie d'élimination des gaspillages attire alors les entreprises occidentales qui cherchent à contrer la concurrence mondialisée. Cet attrait est d'autant plus marqué avec la crise de 2008 pour obtenir des gains rapides [UGH 12]. Théorisation du TPS, le Lean attire aujourd'hui de nombreuses entreprises de tous secteurs et de toutes tailles [WOM 12]. Les résultats rencontrés à court terme par ces entreprises semblent leur donner raison.

\subsection{Des gains opérationnels rencontrés sur le court terme}

En effet, les entreprises qui adoptent tout ou partie des principes ou outils du Lean rencontrent des gains spectaculaires à court terme, c'est-à-dire à moins de douze mois après la mise en place de la démarche [DRE 04]. Ces résultats concernent les aspects dits « opérationnels » de la performance comme, par exemple, l'augmentation de la productivité, la réduction des encours ou bien l'amélioration du respect des délais de livraison. Par performance opérationnelle, nous entendons la performance qui a trait aux opérations, c'est-à-dire aux processus élémentaires qui composent l'activité de production [GIA 03]. Si la performance opérationnelle est effectivement au rendez-vous dans les premiers mois qui succèdent à l'adoption du Lean, plusieurs études rapportent une évolution inattendue de ces mêmes résultats.

\subsection{Une chute des résultats sur le moyen et long terme}

Selon [CAS 09] et [LIK 11], plusieurs entreprises rencontrent une stagnation, voire une chute des résultats initialement atteints avec le Lean. Ce phénomène est observé à moyen ou long terme, c'està-dire environ douze mois après le déploiement de tout ou partie de la démarche Lean dans les entreprises touchées. Dans certains cas, cette chute s'accompagne d'une dégradation de la santé des opérateurs de production [CAR 08]. Ces observations posent question : pourquoi une telle chute des résultats atteints? Comment pallier les limites du Lean?

\subsection{Problématique}

Nous avons choisi d'axer notre recherche sur ces observations et les interrogations qui en découlent. Alors que certains auteurs, tels que [JAG 14] attribuent cette chute à une application incomplète ou désordonnée des principes et outils du TPS par les entreprises lean, d'autres auteurs invoquent des raisons liées à la « logique de fonctionnement» des entreprises lean [PAR 09]. Dans 
nos recherches, nous privilégions cette voie. Nous nous sommes précisément intéressés au fonctionnement des entreprises lean dans leur manière d'évaluer la performance des « actions lean », actions visant à transformer les activités de production pour les rationaliser : quels objectifs sont privilégiés par les entreprises lean ? Quels indicateurs sont utilisés ? Quels leviers d'action sont engagés ? Au sens de [GIR 08], ce questionnement renvoie au «modèle de performance ». Par ce focus sur le modèle de performance adopté par les entreprises lean, notre objectif est de définir un nouveau modèle qui pallie les limites évoquées précédemment (chute des résultats opérationnels, accompagnés ou non d'une dégradation de la santé des opérateurs). Pour cela, nous avons mené des entretiens auprès de dix entreprises lean.

Nous allons décrire notre cheminement depuis les entretiens menés avec dix entreprises lean jusque vers un nouveau modèle de performance qui cherche à pallier les limites du Lean. Dans un premier temps, nous constaterons que le modèle de performance Lean se focalise sur l'efficience opérationnelle. Dans un second temps, nous proposerons de faire évoluer ce modèle. Enfin, nous proposerons un cadre de modélisation qui permet de représenter les impacts de ce nouveau modèle sur la prise de décision.

\section{Un modèle de performance Lean axé sur l'efficience operationnelle}

Pour caractériser le modèle de performance utilisé par les «entreprises Lean », nous avons choisi de mener des entretiens auprès de plusieurs entreprises qui ont déployé tout ou partie des principes et outils du Lean.

\subsection{Méthodologie adoptée pour mener les entretiens}

Nous avons rencontré dix entreprises situées dans le secteur géographique du Pays Basque. Nous avons sélectionné ces entreprises selon la maturité de la démarche Lean mise en place. Nous avons retenu trois prérequis pour choisir ces entreprises. Le premier concerne la formalisation d'un plan d'action Lean. Le second concerne le déploiement de plusieurs principes ou outils Lean. Le dernier concerne la pratique de l'amélioration continue sous la forme de réunions périodiques abordant les problèmes quotidiens générant des gaspillages. Le tableau 1 présente quelques éléments de contexte de ces entreprises.

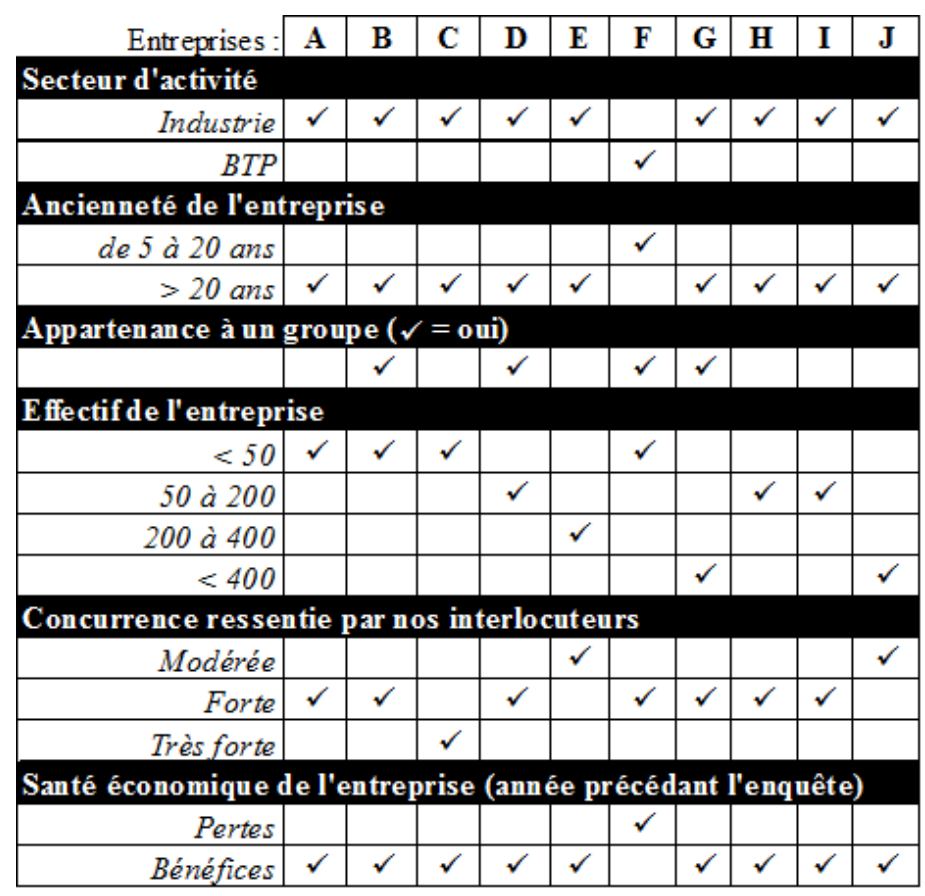

Tableau 1. Description des entreprises Lean approchées 
Nos entretiens ont ciblé des « décideurs » qui occupent un rôle central dans le déploiement de démarche Lean en interne. Dans la majorité des cas, ces décideurs en sont les initiateurs. Dans la totalité des cas, ils ont la responsabilité du déploiement des activités liées à la démarche Lean (exemples : juste à temps, VSM, 5S, réunions périodiques de résolution de problème). Dans le cadre d'un protocole d'entretien formalisé, de type semi directif, nous avons établi un questionnaire composé de questions à la fois ouvertes et fermées. Ces entretiens se sont déroulés sur une durée d'environ deux heures par interlocuteur. Dans le but de comparer et de fiabiliser les réponses apportées, les entretiens ont été renouvelés quelques mois après leur première réalisation.

Notre questionnaire comportait plusieurs thématiques, dont une abordait les principes et outils choisis par l'entreprise questionnée. Pour caractériser les principes et outils du Lean, nous nous sommes appuyés sur la classification apportée par [LIK 04]. Cet auteur classe les principes du TPS (et donc du Lean) en quatre catégories (P1 à P4) que nous synthétisons ainsi :

-P1 - Investir sur le long terme (ex : former les salariés au lieu de distribuer des dividendes),

-P2 - Éliminer les gaspillages dans les activités de production (ex : déploiement du 5S ou du « juste à temps »),

-P3 - Accompagner les opérateurs dans la résolution de problème (ex : les former aux outils, les challenger quotidiennement),

-P4 - Améliorer de manière continue en se basant sur le Kaizen, c'est-à-dire en prenant en compte les contraintes « du terrain » dans l'analyse des problèmes et en cherchant un consensus auprès des personnes impliquées.

Cette typologie nous a permis de comparer les pratiques adoptées par chaque entreprise et d'appréhender le modèle de performance adopté par ces entreprises Lean.

\subsection{Des principes et outils focalisés sur l'efficience opérationnelle}

Selon [GIR 08], un modèle de performance se définit par rapport aux objectifs et aux leviers d'actions privilégiés par les acteurs qui adoptent ce modèle. Pour connaitre le modèle de performance Lean adopté, nous avons donc interrogé plus précisément nos interlocuteurs au sujet :

- des principes et outils du Lean privilégiés (traduisant les « leviers d'action»)

- des indicateurs de performance utilisés (traduisant les « objectifs »).

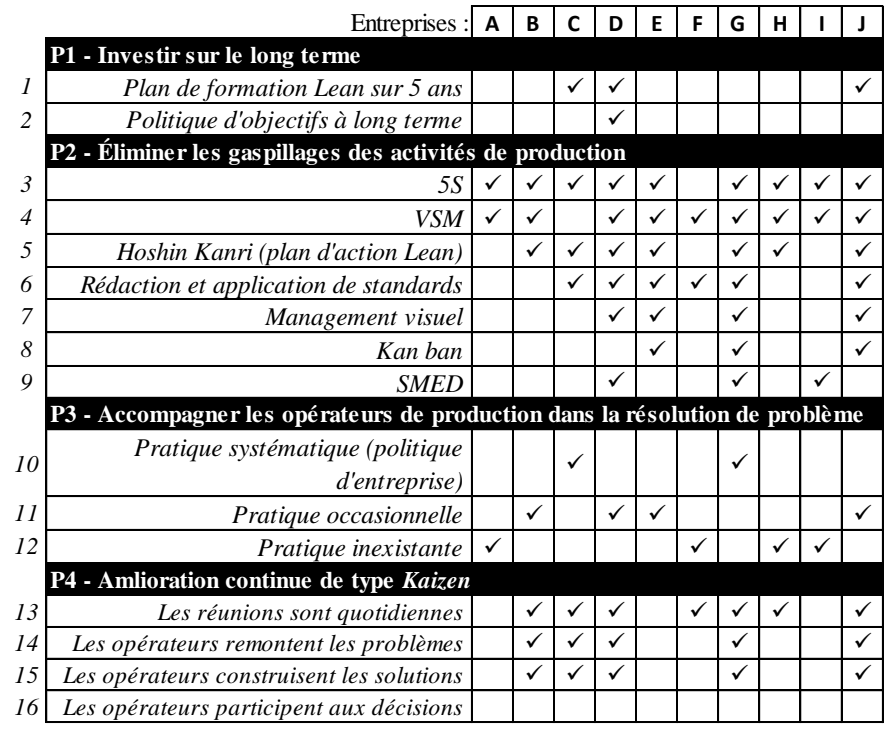

Tableau 2. Résultats de l'enquête sur les principes et outils utilisés pour le déploiement de la démarche Lean 
Concernant le premier point, le tableau 2 apporte une synthèse des pratiques du Lean dans chacune des dix entreprises. Les lignes 3 à 9 montrent un attrait unanime pour la catégorie P2 d'élimination des gaspillages et plus particulièrement pour les outils du 5S et du VSM. Nos interlocuteurs justifient ce choix par la capacité de ces outils à éliminer rapidement les gaspillages, à obtenir des gains opérationnels et à démontrer l'efficacité de la démarche Lean en interne. La ligne 1 du tableau 3, qui synthétise les résultats observés suite au déploiement de la démarche Lean, semble leur donner raison : la totalité des entreprises a rencontré des gains d'ordre opérationnel.

Cependant, le reste du tableau montre que les entreprises Lean ne sont pas ou peu intéressées par les autres principes du Lean : peu d'entreprises investissent dans la formation des salariés ou fixent des objectifs à long terme (principe P1, lignes 1 et 2 du tableau 2), peu incitent et forment les opérateurs à la résolution de problème dans le cadre d'une politique interne de l'entreprise (principe P3, ligne 10 du tableau 2) et peu appliquent le Kaizen lors de l'amélioration continue Lean (principe $\mathrm{P} 4$, lignes 15 et 16 du tableau 2).

Ainsi, le modèle de performance adopté dans les entreprises Lean se caractérise par un choix de leviers d'action visant à éliminer les gaspillages au sein des opérations de production. Ce constat rejoint la position de plusieurs auteurs tels que [WOM 12] et [LIK 04] qui constatent une focalisation des entreprises sur les principes et outils de réduction des gaspillages plutôt que ceux liés au management et à la culture Kaizen. Afin de compléter notre définition du modèle de performance utilisé dans les dix entreprises Lean, nous nous sommes aussi intéressés aux indicateurs de performance utilisés. Les lignes 4 à 12 du tableau 3 présentent les indicateurs utilisés dans les dix entreprises Lean. Il apparait que les indicateurs les plus fréquemment utilisés concernent à la fois les résultats mesurés « en sortie » des opérations de production (ex : délais, rebuts), mais aussi le déroulement du processus lui-même (ex : productivité, rendement).

$\mathrm{Au}$ regard des leviers d'action privilégiés (éliminer les gaspillages) et des indicateurs fréquemment utilisés (de type résultat et processus), nous constatons que nos interlocuteurs adoptent un modèle de performance privilégiant l'efficience opérationnelle des activités de production, c'està-dire la recherche du meilleur rapport entre les résultats atteints et les moyens mobilisés pour y parvenir (ex : respecter les délais avec un minimum de stock ou de temps passé).

\begin{tabular}{|c|c|c|c|c|c|c|c|c|c|c|c|}
\hline & Entreprises : & $\mathbf{A}$ & B & C & D & E & $\mathbf{F}$ & G & H & I & $\mathbf{J}$ \\
\hline \multicolumn{12}{|c|}{ Résultats après 12 mois de mise en œuvre de la démarche Lean } \\
\hline 1 & Hausse des résultats opérationnels & $\checkmark$ & $\checkmark$ & $\checkmark$ & $\checkmark$ & $\checkmark$ & $\checkmark$ & $\checkmark$ & $\checkmark$ & $\checkmark$ & $\checkmark$ \\
\hline 2 & Dégradation de la santé des opérateurs & & & $\checkmark$ & & & & $\checkmark$ & $\checkmark$ & $\checkmark$ & \\
\hline 3 & Rejet de la démarche, passivité des opérateurs & $\checkmark$ & $\checkmark$ & $\checkmark$ & & $\checkmark$ & $\checkmark$ & $\checkmark$ & $\checkmark$ & $\checkmark$ & $\checkmark$ \\
\hline \multicolumn{12}{|c|}{ Indicateurs et fréquence $(q=$ quotidien, $h=$ hedomadaire, $\mathbf{m}=$ mensuel, $\mathbf{a}=$ annuel) } \\
\hline 4 & Taux de service, taux de respect des délais & $\mathrm{q}$ & $\mathrm{q}$ & & $\mathrm{h}$ & & $\mathrm{h}$ & $\mathrm{q}$ & $\mathrm{q}$ & & q \\
\hline 5 & Nombre de non-conformité, rebuts & & q & $\mathrm{h}$ & $\mathrm{q}$ & $\mathrm{h}$ & & q & & $\mathrm{h}$ & $\mathrm{q}$ \\
\hline 6 & Productivité, rendement, "efficience" & & & $\mathrm{h}$ & q & & $\mathrm{h}$ & $\mathrm{q}$ & & $\mathrm{h}$ & $\mathrm{q}$ \\
\hline 7 & Production réalisée & & & & $\mathrm{q}$ & & $\mathrm{m}$ & $q$ & $q$ & $\mathrm{~h}$ & \\
\hline 8 & Taux de Rendement Synthétique (TRS) & & & & q & $\mathrm{h}$ & & $\mathrm{m}$ & & & \\
\hline 9 & Temps d'écoulement & & & & $q$ & & & $\mathrm{q}$ & & & \\
\hline 10 & Accidents et maladies professionnelles & $\mathrm{a}$ & $\mathrm{a}$ & $\mathrm{a}$ & & $\mathrm{a}$ & $\mathrm{a}$ & $\mathrm{q}$ & & $\mathrm{h}$ & $q$ \\
\hline 11 & Turn over & & & & & $\mathrm{a}$ & & & & & \\
\hline 12 & Absentéisme & a & $\mathrm{a}$ & a & & $\mathrm{a}$ & a & $\mathrm{m}$ & & $\mathrm{h}$ & $q$ \\
\hline
\end{tabular}

Tableau 3. Résultats de l'enquête sur les effets de la démarche Lean adoptée et les indicateurs utilisés

Cependant, un autre constat attire notre attention : les indicateurs les plus fréquemment utilisés portent essentiellement sur la dimension technique des moyens engagés (cf. lignes 4 à 9 du tableau 3 ) et portent peu sur la dimension humaine (cf. lignes 10 à 12 du tableau 3). Lorsque la dimension humaine est mesurée, les indicateurs «humains »sont mesurés sur un terme très éloigné de 
l'évènement considéré (les $2 / 3$ des indicateurs sont mesurés une fois par an), ce qui ne favorise pas l'intégration de cette dimension dans l'évaluation de l'efficience. Nous retrouvons cette caractéristique au travers des modèles de performance proposés par plusieurs auteurs, modèles destinés à mesurer les effets du Lean. Par exemple, [BHA 08] propose des indicateurs de «process » (ex : temps de cycle, rotation des stocks) mais aussi humains (« people », ex : accidents, absentéisme, satisfaction des salariés, taux de maintien des salariés à leur poste).

Ainsi, au regard des leviers d'action privilégiés et des objectifs visés, il apparait que nos interlocuteurs adoptent un modèle de performance qui privilégie l'efficience des opérations «techniques»de production. Ceci se fait au détriment de la dimension humaine qui est sousconsidérée en raison de l'éloignement du terme auquel les indicateurs humains sont mesurés. Il s'agit ici d'une limite importante du modèle utilisé : en effet, comment évaluer la réelle performance d'une action Lean si certains effets latents ne sont pas pris en compte ? Nous allons constater que cette considération est le reflet d'une vision précise que nos interlocuteurs portent sur la place occupée par les opérateurs dans la performance de l'entreprise.

\subsection{Une efficience opérationnelle éloignée de l'humain}

Durant nos entretiens, nous avons cherché à appréhender les représentations mentales de nos interlocuteurs au sujet de la dimension humaine de la performance. Pour cela, nous avons inclus dans nos entretiens des questions ouvertes telle que «Que devrait faire l'entreprise pour que les opérateurs de production soient performants dans leur travail ?». Le tableau 4 montre que, selon nos interlocuteurs, la performance des opérateurs passe par la définition des «bonnes » procédures de travail (ligne 1 du tableau 4), la mise en œuvre de contrôles fréquents (ligne 2 du tableau 4) et la formation des opérateurs à ces procédures (ligne 3 du tableau 4).

\begin{tabular}{|c|c|}
\hline $\begin{array}{l}\text { Réponses données à la question : } \\
\text { "Que devrait faire une entreprise pour } \\
\text { que les opérateurs de production } \\
\text { soient performants dans leur travail ?" }\end{array}$ & $\begin{array}{l}\text { Taux de réponse } \\
\text { dans les dix } \\
\text { entreprises Lean }\end{array}$ \\
\hline Définir les bonnes procédures & $70 \%$ \\
\hline Réaliser des contrôles fréquents & $70 \%$ \\
\hline Former aux bons comportements & $60 \%$ \\
\hline Récompenser les opérateurs & $30 \%$ \\
\hline
\end{tabular}

Tableau 4. Résultats de l'enquête sur les représentations mentales adoptées par nos interlocuteurs

Pour favoriser le respect des procédures de travail, certains de nos interlocuteurs estiment que la mise en place de primes peut influencer (ligne 4 du tableau 3). Ainsi, la performance passerait par le respect strict de procédures adaptées et communes dans un environnement de travail déterminé.

Ces éléments suggèrent que nos interlocuteurs adoptent une vision qui considère que la performance peut être atteinte si les opérateurs de production agissent comme des "exécutants ", c'est-à-dire qu'ils évoluent dans un environnement dans lequel ils doivent se conformer à des procédures («contrôles fréquents ») qui ont été pensées pour eux (les «bonnes procédures »). De notre point de vue, cette vision est réductrice du rôle que les opérateurs de production jouent dans la performance de l'entreprise, notamment lorsqu'ils font face aux aléas de production. À l'instar de [PIL 07], nous proposons d'adopter une vision qui considère que les opérateurs de production 
occupent un rôle « acteur » dans leur environnement de travail et donc dans la performance de l'entreprise.

\section{Un modèle de performance qui intègre la vision « acteur » de l'humain au travail}

\subsection{Un nouveau regard sur l'activité de travail}

Adopter une vision « acteur » implique un regard différent sur la manière dont les opérateurs de production réalisent les tâches (ou procédures) de travail qui leur sont assignées. Dans le domaine scientifique de l'ergonomie de l'activité de travail, il est considéré qu'il existe toujours un écart entre le travail prescrit (ex : procédures, standards) et le travail réalisé. Cet écart tient d'abord du fait qu'il est impossible pour un décideur de prévoir à l'avance comment le travail doit être réalisé en raison des variabilités liées au contexte (ex : variation de la qualité d'une matière à transformer) et en raison des variabilités inter et intra individuelles (ex : différence des compétences d'un individu à l'autre). Cet écart « prescrit /réel » tient aussi du postulat qui considère que les situations de travail ne peuvent jamais être stabilisées (ex : absence totale de panne, matière première de qualité constante). L'élimination totale des Mura (irrégularités de production) ne peut pas être atteinte, et un opérateur de production sera confronté tôt ou tard à une situation variable ou inattendue [BOU 10]. Du fait de ces variabilités et des objectifs de production fixés par l'entreprise, les opérateurs de production sont amenés à " réguler », c'est-à-dire créer des modes opératoires singuliers (parfois inconscients) pour atteindre les résultats demandés tout en préservant leur état interne (ex : fatigue, douleur). La figure 1 présente ce mécanisme de régulation [GUE 01].

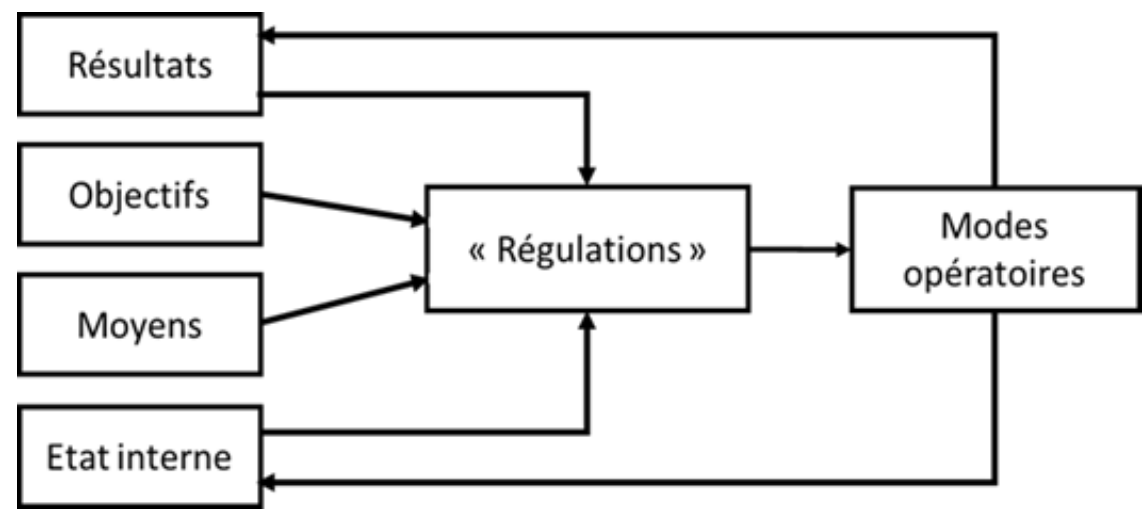

Figure 1. "Modèle de régulation de l'activité de travail » [GUE 01]

Ce mécanisme suggère que les opérateurs déploient des «tours de main » (modes opératoires) qui leur permettent de déjouer les variabilités qui se présentent et d'atteindre les résultats attendus tout en préservant leur santé. Cette capacité à créer des modes opératoires pertinents va dans le sens d'une vision " acteur » qui considère que les opérateurs sont bien plus que de simples exécutants : ils participent ainsi « activement » à la performance de l'entreprise et, dans le cas du Lean, à l'efficience opérationnelle. Cependant, pour permettre aux opérateurs de production d'adopter des modes opératoires pertinents, il est nécessaire que l'environnement de travail offre les marges de manœuvre nécessaires [BAD 14]. Cela implique une réflexion interne systématisée portant sur les marges de manœuvre qu'il est nécessaire de prévoir lors de la mise en place de toute action d'élimination des gaspillages (action Lean). Également, ceci implique de porter un autre regard sur la manière d'évaluer la performance d'une action Lean préalablement engagée, c'est à dire la manière d'évaluer l'efficience opérationnelle. Porter un autre regard sur l'efficience opérationnelle, c'est faire évoluer sa propre vision sur les résultats et les moyens à prendre en compte. Nous allons décrire en quoi le mécanisme de régulation de l'activité de travail permet d'enrichir ce regard sur les résultats et les moyens à prendre en compte. 


\subsection{Une nouvelle manière de considérer les résultats obtenus et les moyens mobilisés}

Pour représenter cette nouvelle manière de considérer les résultats et les moyens, nous allons nous appuyer sur le modèle proposé par [BES 93] que nous nommerons le « triangle de gestion » (cf. figure 2). Ce triangle met en évidence trois manières de considérer la performance d'une action donnée :

-l'efficacité, qui apprécie si les résultats atteints sont bien conformes aux objectifs initiaux,

-l'efficience, qui apprécie si les résultats sont atteints avec l'engagement le plus faible des moyens,

-la pertinence, qui apprécie si les moyens choisis sont adaptés aux objectifs fixés.

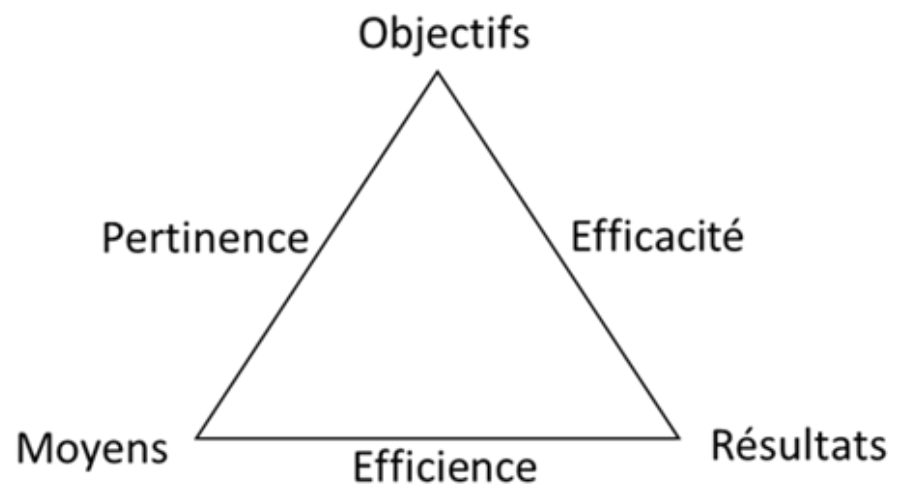

Figure 2. «Les problématiques clés » [BES 93]

Nous représentons d'abord le modèle de performance Lean adopté par les entreprises Lean au moyen du «plan de l'efficience opérationnelle Lean » de la figure 3. Ce plan comporte les trois sommets du triangle avec les correspondances suivantes : les objectifs correspondent aux objectifs d'élimination des gaspillages (c'est le but du Lean), les résultats considérés sont d'ordre technique et opérationnel (cf. indicateurs décrits dans le tableau 2) et les moyens considérés sont le déploiement des principes et outils du Lean (cf. leviers d'action décrits dans le tableau 2).

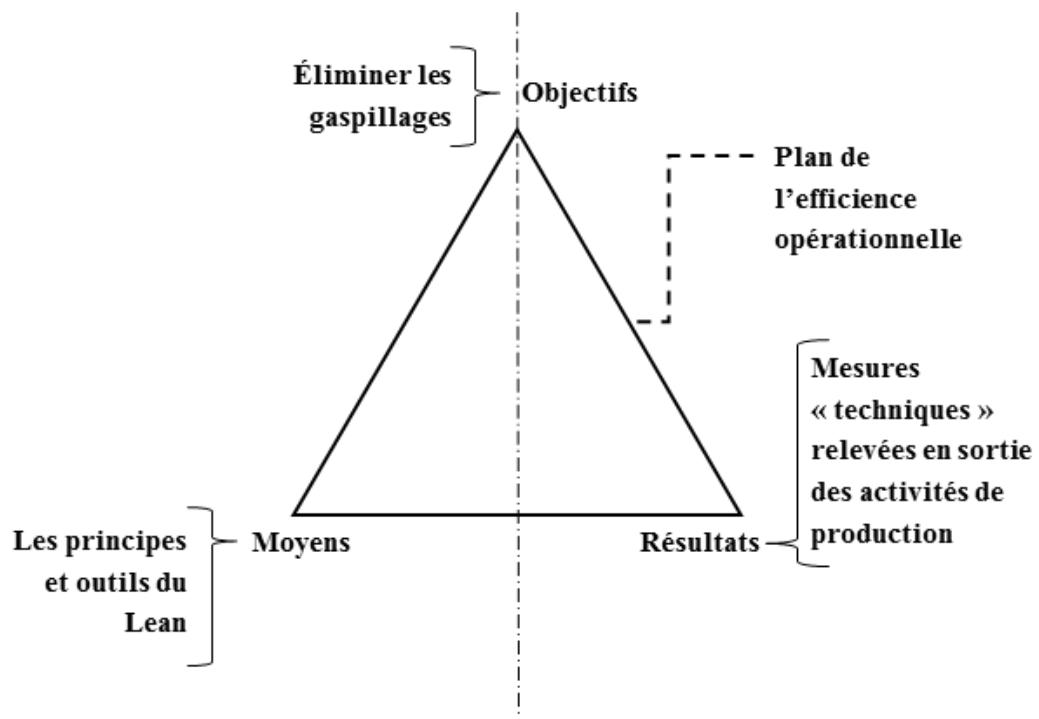

Figure 3. Plan de l'efficience opérationnelle Lean 
Avec une vision « acteur » telle que présentée dans la figure 4, l'efficience est abordée sous un aspect qui intègre la dimension humaine de la performance via le mécanisme de régulation de l'activité de travail. Nous représentons cette approche de la performance avec le « plan de l'Efficience Humaine ». Ce plan comporte les trois sommets du triangle avec les correspondances suivantes :

- concernant les résultats, il ne s'agit plus seulement de se focaliser sur la mesure des résultats techniques relevés en sortie des processus, mais aussi de s'intéresser à l'état interne des individus suite à l'obtention de ces résultats (ex : quelle fatigue ou douleur ressentie en fin de journée suite à tel aménagement du Lean ?);

- de même, avec une vision « acteur ", il ne s'agit plus de seulement de considérer que les résultats ont été obtenus au moyen du seul déploiement des «bonnes » actions Lean, mais aussi par la création et le déploiement des modes opératoires par les opérateurs de production lors de l'apparition des variabilités contextuelles et inter / intra individuelles inhérentes au quotidien de travail.

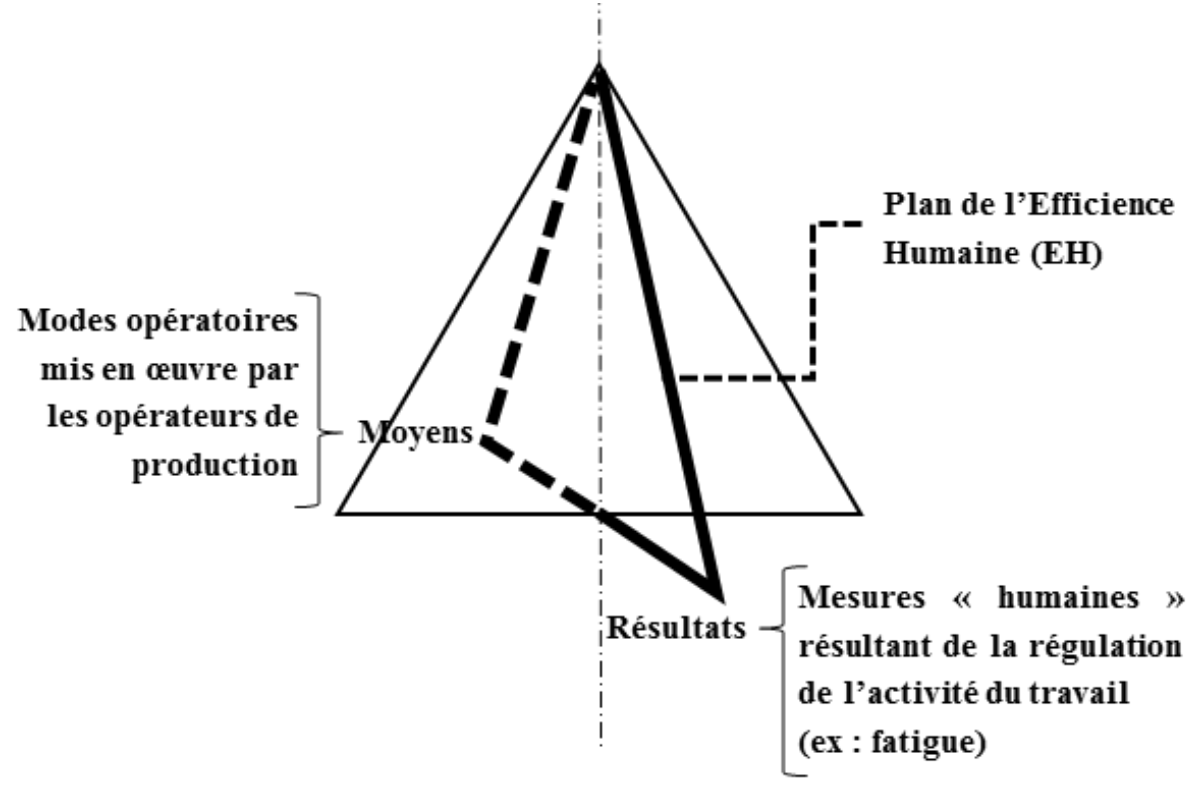

Figure 4. Plan de l'efficience humaine Lean EH

En nous appuyant sur la vision « acteur », nous proposons ainsi un modèle de performance qui se base sur une complémentarité entre l'approche de l'efficience opérationnelle et l'approche de l'efficience humaine, telle que nous l'avons définie avec le mécanisme de régulation de l'activité de travail. Nous nommons ce modèle le « modèle de performance Lean $\mathrm{EH}$ » $\mathrm{EH}$ pour Efficience Humaine).

Ce double regard permet d'évaluer la performance des actions Lean sous plusieurs facettes. Il permet précisément de mesurer les effets latents d'une action Lean qui, malgré des gains initialement prometteurs, peuvent dégrader la santé des salariés à moyen-long terme et impacter les gains initialement obtenus. Cette évaluation de la performance a posteriori d'une action Lean peut alimenter une base de connaissances permettant d'identifier a priori des points de vigilance sur l'application de certaines actions Lean au regard des effets mesurés. Ainsi, lors de l'utilisation du VSM (outil de diagnostic a priori de certaines actions Lean), les utilisateurs peuvent enrichir leur réflexion et constater, par exemple, que certains processus, apparemment « gaspilleurs », se révèlent être, au contraire, des soutiens pour la régulation de l'activité de travail des opérateurs de production (marges de manœuvre). Comme nous l'avons évoqué précédemment, cette manière enrichie d'aborder l'efficience implique une réflexion interne systématisée portant sur les marges de 
manœuvre qu'il est nécessaire de prévoir lors de la mise en place de toute action d'élimination des gaspillages (action Lean). Ceci implique des changements dans les pratiques quotidiennes et sur la prise de décision (ex : réfléchir collectivement sur les marges de manœuvre; mesurer les effets de l'absence de ces marges sur l'état interne des opérateurs). Pour caractériser les impacts du modèle de performance Lean EH sur la prise de décision, il est nécessaire de définir un cadre de modélisation adapté à la vision « acteur».

\section{Un cadre de modélisation pour représenter les impacts du Lean EH}

Pour représenter la prise de décision, nous allons nous appuyer sur la méthode GRAI (Graphe de Résultats et Activités Inter reliées). Cette méthode permet de modéliser le système décisionnel, informationnel et physique d'une entreprise. Elle comprend un cadre de modélisation, des outils de modélisation et une méthodologie [DOU 84].

\subsection{Une évolution de GRAI pour représenter les impacts}

GRAI comprend des outils de modélisation (la grille GRAI en l'occurrence pour la formalisation du système décisionnel) pour représenter de manière détaillée la prise de décision entre deux centres de décision au moyen d'un cadre de décision qui comprend les éléments suivants [DOU 84] :

-les objectifs, qui décrivent les performances attendues,

- les indicateurs de performance, choisis au regard de ces objectifs,

- les variables d'action, qui décrivent ce sur quoi le centre de décision concerné peut agir,

- les critères, qui aident au choix parmi les variables d'action,

-les contraintes, qui décrivent les limites du potentiel des variables d'action.

Ces éléments de modélisation sont appropriés pour représenter les interactions entre un centre de décision, qui décide et qui prescrit, et un autre centre de décision de niveau inférieur, qui serait piloté par le premier via un tel cadre de décision.

Dans un contexte de production Lean EH, c'est-à-dire dans lequel les salariés échangent entre eux au sujet des marges de manœuvre, le formalisme GRAI ne permet pas de retranscrire de manière détaillée toutes les interactions liées à cette thématique : ces interactions ont lieu au sein du « système physique » et certaines sont de nature à remettre en cause la caractérisation des prises de décision dont dépend le système physique. Dans ce contexte, le centre de décision qui est en contact avec le système physique (par exemple un groupe d'opérateurs de production), et qui le pilote, applique lui-même les consignes portées dans le cadre de décision, alors que dans un contexte Lean, nous souhaitons lui conférer plutôt un rôle « acteur », ainsi il serait susceptible d'apporter lui-même des évolutions dans les mécanismes de prise de décision qui le concernent, ce qui revient à faire évoluer la structuration de la grille GRAI.

Nous proposons de faire évoluer le formalisme GRAI en nous appuyant sur le modèle GRAI R\&D, [GIR 99] et [MER 14], qui met en interaction un centre de décision (qui conduit) et un centre de conception (conduit, mais « acteur »). Ce dernier est chargé de proposer des solutions de conception de produit et se trouve en contact avec le système technologique (i.e. le système " physique » en conception), c'est-à-dire avec l'environnement propre à la conception des produits dans l'entreprise. Dans GRAI R\&D, le centre de décision émet un cadre de conception à destination du centre de conception. Ce cadre est composé des éléments suivants :

- les objectifs de conception, qui traduisent ce que le centre de conception doit atteindre,

-les objectifs de performance, qui évaluent périodiquement les activités du centre, 
- les moyens attribués (moyens financiers, ressources humaines, physiques et informationnelles),

-le domaine de compétence.

Ces éléments permettent de représenter plus finement le caractère « acteur » du système conduit avec, par exemple, la possibilité d'ajuster les moyens à disposition ou bien les compétences nécessaires au regard du caractère variable des situations rencontrées.

Nous allons nous appuyer sur GRAI R\&D, et plus particulièrement de son cadre et centre de conception, pour représenter la prise de décision dans un système de production Lean EH. Pour cela, nous distinguons deux caractéristiques essentielles d'un système de production Lean : la conduite de la production proprement dite et la conduite de l'amélioration continue. Nous proposerons ainsi deux modèles que nous nommerons « les modèles GRAI Lean EH ».

\subsection{Modélisation de la conduite de la production}

La conduite de la production renvoie à la conduite du système physique par le système décisionnel, et donc à la mise en œuvre des routines de production telles que, par exemple, la réception d'une commande, la rédaction d'un plan d'ordonnancement et l'organisation pour répondre à cette commande.

En contexte Lean, l'organisation est modifiée par des actions d'élimination des gaspillages (actions Lean), décidées dans un plan d'action annuel ou pluri annuel. Ce plan d'action est construit dans le cadre de l'application d'un outil emblématique du Lean, nommé Hoshin Kanri, qui consiste à définir des orientations de la démarche Lean, déployer les objectifs, aligner les ressources nécessaires et planifier des projets pour l'année courante [BYR 14].

En contexte Lean $\mathrm{EH}$, la vision « acteur » suggère que les différents centres de décision règlent l'organisation de manière conjointe en menant une réflexion sur les marges de manœuvre. À l'aide de GRAI R\&D nous pouvons représenter en figure 5 un centre de décision assurant la conduite d'un sous-ensemble du physique, sous-ensemble que nous nommons «centre opérant». La mission de ce centre opérant est de produire, c'est à dire de transformer des matières premières et/ou des produits intermédiaires en produits finis ou d'autres produits intermédiaires. Pour cela, il crée et déploie des modes opératoires pertinents, comme décrit avec le mécanisme de régulation de l'activité de travail.

Le centre de décision qui conduit le centre opérant émet vers lui un « cadre de travail » qui comprend les éléments suivants :

-les « objectifs de performance », qui fixent les objectifs à atteindre (ex : produire 10 pièces bonnes par heure),

- les « objectifs de production », qui décrivent le mode opératoire «prescrit » (ex : utiliser tel outil dans tel ordre de la gamme de fabrication)

- les « moyens attribués », qui décrivent les marges de manœuvre sur lesquelles le centre opérant peut s'appuyer pour faire face aux différentes variabilités rencontrées lors de la production. Ces éléments sont issus des réflexions internes, comme décrit précédemment (ex : liberté de choix entre tel ou tel outil, tel ou tel ordre de tâche)

-le « domaine de compétence », qui décrit les compétences que le centre opérant doit avoir pour réaliser la production.

Ces éléments cadrent ainsi le travail réalisé par le centre opérant en intégrant une réflexion sur les marges de manœuvres nécessaires qui permet au centre opérant d'adopter des modes opératoires pertinents. Les impacts de la vision « acteur » sur la prise de décision, d'un centre de décision à un 
centre opérant, peuvent ainsi être représentés dans le détail, du moins pour les activités liées à la conduite de la production.

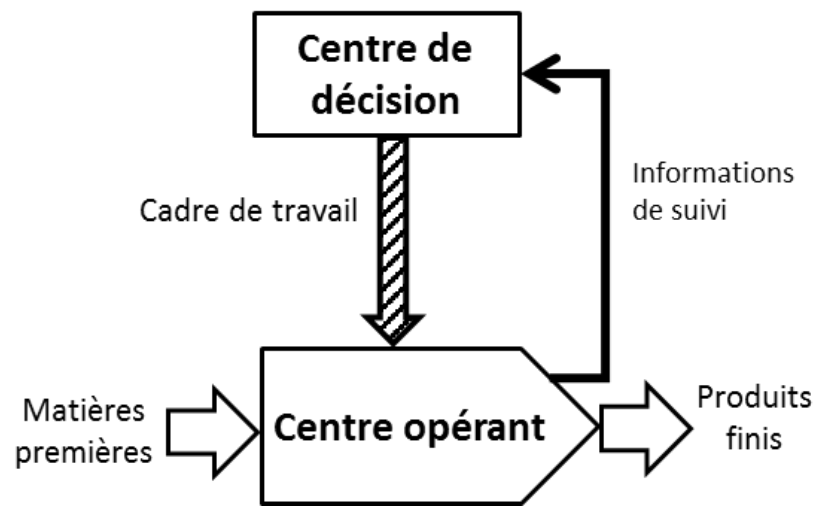

Figure 5. Modèle GRAI Lean EH pour la conduite des activités de production

Nous allons maintenant proposer un modèle qui permet de représenter les impacts de la vision « acteur » sur la conduite des activités liées à l'amélioration continue.

\subsection{Modélisation de l'amélioration continue}

Dans le paragraphe 3.1, nous avons décrit que les entreprises Lean adoptent l'amélioration continue, c'est-à-dire des activités de résolution de problème destinées à éliminer continuellement les gaspillages. Ces activités se déroulent en parallèle de la conduite de la production et ont pour effet de transformer en permanence l'organisation du travail, à une fréquence plus élevée et plus localisée que la transformation issue du déploiement du Hoshin Kanri. La résolution de problème peut prendre plusieurs formes dans une entreprise Lean. La plus commune est la tenue de groupes internes de résolution de problème associant des managers de différents niveaux et des opérateurs. Ces groupes ont pour mission de concevoir des solutions aux problèmes remontés par les opérateurs et de décider leur mise en œuvre.

En contexte Lean, les résultats de notre enquête montrent que ces groupes de résolution de problème disposent de marges de liberté restreintes pour identifier des solutions. La nature des solutions doit se «conformer » aux orientations fixées par le Hoshin Kanri, c'est à dire suivre de près les principes d'élimination de gaspillages (ex : éliminer les stocks, produire pièce à pièce, réduire les mouvements). Cette emprise des principes d'élimination des gaspillages, que nous nommons ci-après la «prédétermination Lean », confère aux groupes de résolution de problème un rôle restreint, davantage « exécutant».

En contexte Lean $\mathrm{EH}$, la vision « acteur » suggère que les groupes de résolution de problème sont les plus à même de proposer des solutions pertinentes, même si celles-ci doivent, dans certains cas, contredire les principes de l'élimination des gaspillages. À l'aide de GRAI R\&D, nous souhaitons être capable de représenter les activités d'un groupe de résolution de problème, y compris dans sa relation avec le centre de décision qui pilote un (ou plusieurs) centre(s) opérant(s) confronté à ce problème. À travers le rôle « acteur » qu'il est nécessaire de considérer à ce groupe de résolution, notre but est de caractériser ensuite comment certaines solutions remettant en cause la structuration de la prise de décision (via la grille GRAI) peuvent être prises en compte pour effectuer cette transformation du système décisionnel, à une échelle locale plus ou moins étendue. Nous nommons ce groupe de résolution un « centre de transformation Lean $\mathrm{EH} »$ (Figure 6). La mission de ce centre de transformation Lean EH est de concevoir les solutions d'organisation du travail les plus appropriées afin d'éliminer les gaspillages venant des problèmes quotidiens rencontrés par les 
opérateurs de production, et remontés par le centre de décision qui conduit ce centre de transformation.

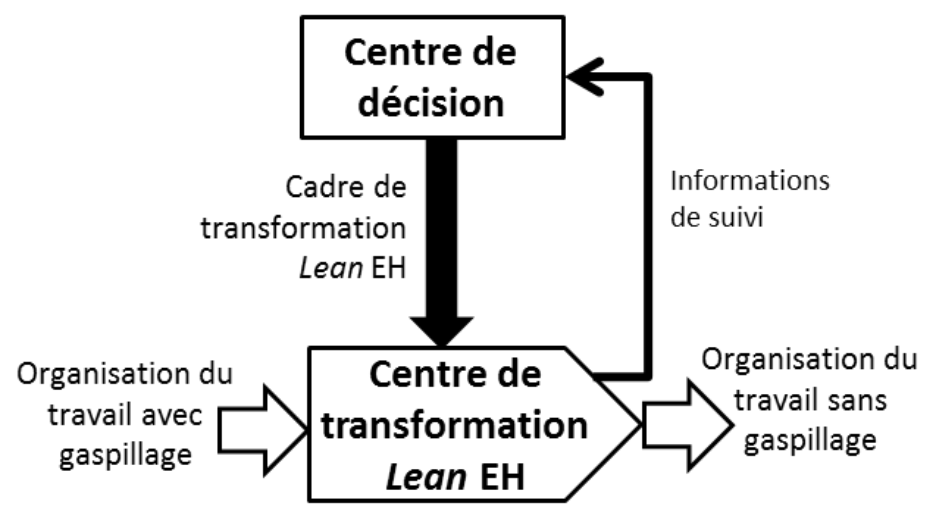

Figure 6. Modèle GRAI Lean EH pour la conduite de l'amélioration continue

Le centre de décision qui conduit le centre de transformation Lean EH émet un « cadre de transformation Lean $\mathrm{EH} »$ qui comprend les éléments suivants :

- les « objectifs de transformation », qui décrivent les objectifs fixés par le centre de décision et que la solution conçue doit atteindre (ex : anticiper et réduire les pannes machine sur tel secteur),

-les « objectifs de performance », qui décrivent le processus de résolution lui-même (ex : trouver une solution en 3 jours),

- les « moyens attribués », décrivant les moyens alloués à la résolution de problème (ex : intégrer tels opérateurs au groupe de réflexion, possibilité de déroger partiellement à telle orientation issue du plan Hoshin Kanri),

-le « domaine de compétence », qui décrit les compétences attendues pour concevoir les solutions.

Ces éléments permettent de cadrer le processus de résolution de problème en intégrant la possibilité d'ajuster les marges de liberté et les moyens alloués au regard de chaque situation rencontrée. Ainsi, les impacts de la vision « acteur » sur la prise de décision peuvent être représentés.

\section{Modélisation du système de production Lean EH : cas d'étude}

Les deux modèles peuvent être regroupés afin de représenter la prise de décision propre à un système de production Lean EH. Dans ce système, les activités de conduite de production et de résolution de problème se déroulent en parallèle. Elles interagissent en particulier sur la question des marges de manœuvre nécessaires à la réalisation de l'activité de travail des opérateurs de production.

\subsection{Présentation du cas d'étude}

Pour illustrer l'application du cadre de modélisation GRAI Lean EH, nous nous référons à une étude de cas réalisée dans l'une des entreprises Lean qui a répondu à notre enquête, que nous nommerons l'entreprise X.

L'entreprise X évolue dans le secteur de la métallurgie. Son activité consiste à assembler des produits finis à partir de divers composants. Pour faire face à une concurrence pressante, l'entreprise a adopté et déployé la démarche Lean afin précisément de réduire les coûts et de fiabiliser les délais 
de livraison. Plusieurs principes et outils du Lean ont été déployés, tels que le 5S, les standards de travail, le Kan Ban et les réunions périodiques de résolution de problème. Un an après la mise en place de la démarche, des problèmes de santé liés aux Troubles Musculo Squelettiques (TMS) sont apparus chez plusieurs opérateurs de l'entreprise. La situation que nous allons décrire pour illustrer l'applicabilité de nos modèles concerne un atelier d'assemblage final. Dans cet atelier, plusieurs opérateurs assemblent des composants pour alimenter deux zones d'expédition qui se différencient par leur degré d'urgence. Avant la démarche Lean, les composants entrants étaient stockés dans une zone d'approvisionnement. Avec la démarche Lean, les composants sont livrés « au compte-goutte » en fonction des commandes reçues. C'est en effet l'application du principe Lean du juste à temps qui vise à réduire les stocks, car considérés comme gaspillages (capital immobilisé).

Quand les composants sont de bonne qualité, l'organisation se déroule comme prévu. Les objectifs de respect de livraison sont atteints. Cependant, lorsqu'un composant fait défaut (ex : pièce mal ajustée, rayure), la production se trouve bloquée. En effet, avec un approvisionnement au compte-goutte, les opérateurs ne disposent plus de pièces d'avance pour remplacer les pièces défectueuses. Ceci pose particulièrement problème lorsqu'une commande urgente se présente. Pour pallier ce problème et ainsi respecter les objectifs de livraison, les opérateurs sont alors amenés à se déplacer dans la zone d'expédition «peu urgentes » et démonter un produit déjà assemblé pour prélever un composant de remplacement. Cette situation a pour inconvénient de générer des tâches supplémentaires (démontage et remontage), réalisées dans l'urgence (facteur de TMS) et d'augmenter la pénibilité au travail en raison de l'exiguïté de la zone d'expédition. Malgré son caractère problématique, cette situation n'a jamais été abordée lors des réunions périodiques de résolution de problème. Le partage d'une vision « exécutante » entre les «décideurs » de l'entreprise dissuade les opérateurs de faire part de ce problème à leur propre chef d'équipe.

\subsection{Modélisation GRAI Lean EH pour la conduite}

Nous décrivons maintenant comment les modèles GRAI Lean EH peuvent s'appliquer à cette situation afin de représenter les impacts d'une vision " acteur ", partagée par l'ensemble des salariés, sur la prise de décision de l'entreprise X (Figure 7). Les opérateurs de production sont représentés par le centre opérant (1). En effet, ce sont eux qui créent et déploient les modes opératoires adaptés pour faire face aux variabilités et contraintes qui se présentent (ici, respecter les délais malgré les composants non conformes dans une organisation en juste à temps).

Les actions du chef d'équipe amenant à ses prises de décision sont représentées par l'intermédiaire du centre de décision (2) qui pilote le centre opérant (1).

Avec une vision « acteur » de l'humain au travail, le chef d'équipe émet un cadre de travail (3) qui fixe comme "objectifs de performance » le respect des délais de livraison avec le moins de temps passé, mais aussi l'objectif de préserver l'état de santé des opérateurs (ex : prévenir la fatigue, réduire le stress). Il fixe des «objectifs de production » qui décrivent non seulement les modes opératoires à adopter en situation normale (ici, assembler des produits avec une livraison au comptegoutte), mais aussi en situation dégradée (prélever un composant au moyen du démontage de produits finis en zone d'expédition). Il fixe comme " moyens » non seulement un système d'approvisionnement au compte-goutte synchronisé aux commandes, mais aussi, par exemple, une implantation de la zone des expéditions afin de permettre aux opérateurs de réaliser le démontage dans les meilleures conditions. Enfin, il fixe comme «domaine de compétences » la nécessité de disposer d'opérateurs qui sachent non seulement assembler les produits, mais aussi, par exemple, démonter et extraire un composant dans un produit fini sans détériorer les composants adjacents.

Lors des réunions quotidiennes de production, les opérateurs font remonter des informations (4) non seulement sur l'indicateur de taux de livraison réalisé, mais aussi sur un indicateur de ressenti 
de fatigue par exemple. La lecture de ces indicateurs donne naissance à une réflexion commune sur les marges de manœuvre nécessaires pour améliorer la situation.

\subsection{Modélisation GRAI Lean EH pour l'amélioration continue}

Prenons l'exemple où ces réflexions conduisent à identifier un problème de configuration de la zone d'expédition provoquant des manutentions pénibles lors du démontage des produits finis.

Au regard de ce problème de configuration, le chef d'équipe et les opérateurs se mettent d'accord pour constituer un groupe de travail destiné à configurer la zone d'expédition. Ce groupe est représenté par le centre de transformation Lean $\mathrm{EH}$ (5). En ce sens, le centre de décision (2) émet un cadre de transformation Lean EH (6) vers le centre de transformation Lean EH (5). Avec la vision « acteur », le centre de décision fixe comme « objectif de transformation » non seulement de pouvoir démonter un produit rapidement, mais aussi de pouvoir le faire avec le minimum de manutention. Il fixe comme « objectif de performance » le fait que le groupe de participants conçoive une solution en moins de deux semaines. Il fixe dans les «moyens attribués » une capacité de décision (marge de manœuvre) sur un périmètre identifié, par exemple ici la possibilité de déroger, lors de la conception de la solution, à certaines orientations fixées par le plan Hoshin Kanri de l'entreprise. Concrètement, les membres du centre de transformation Lean ont la possibilité de ne pas forcément rapprocher deux zones de travail afin d'éviter des risques avérés de coactivité. En ce sens, la Prédétermination Lean est modérée. Il fixe comme « domaine de compétence » non seulement le recours à un responsable logistique, pour valider par exemple le dimensionnement des flux, mais aussi aux opérateurs de production afin de valider des aspects très concrets de l'activité de travail lors de tests.

En retour, en tant qu'information de suivi (7), le centre de transformation Lean EH (5) renvoie au centre de décision (2) une proposition de solution (ici, nouvelle configuration de la zone d'expédition).

Le centre de décision (2) valide et communique (informations de suivi (8)) cette solution au centre de décision $\mathrm{N}+1(9)$.

Prenons ici l'exemple d'un responsable de production qui est représenté par le centre de décision $\mathrm{N}+1$ (9). Ce dernier établit chaque année un plan de production (pour la conduite des activités de production) et un plan Hoshin Kanri (pour le déploiement des actions Lean). Avec une vision " acteur », ce centre de décision (9) révise le cadre de décision (10) émis vers le centre de décision (2). Il fixe non seulement des objectifs de respect des délais, mais aussi des objectifs de réduction de la pénibilité pour l'ensemble des opérateurs de production.

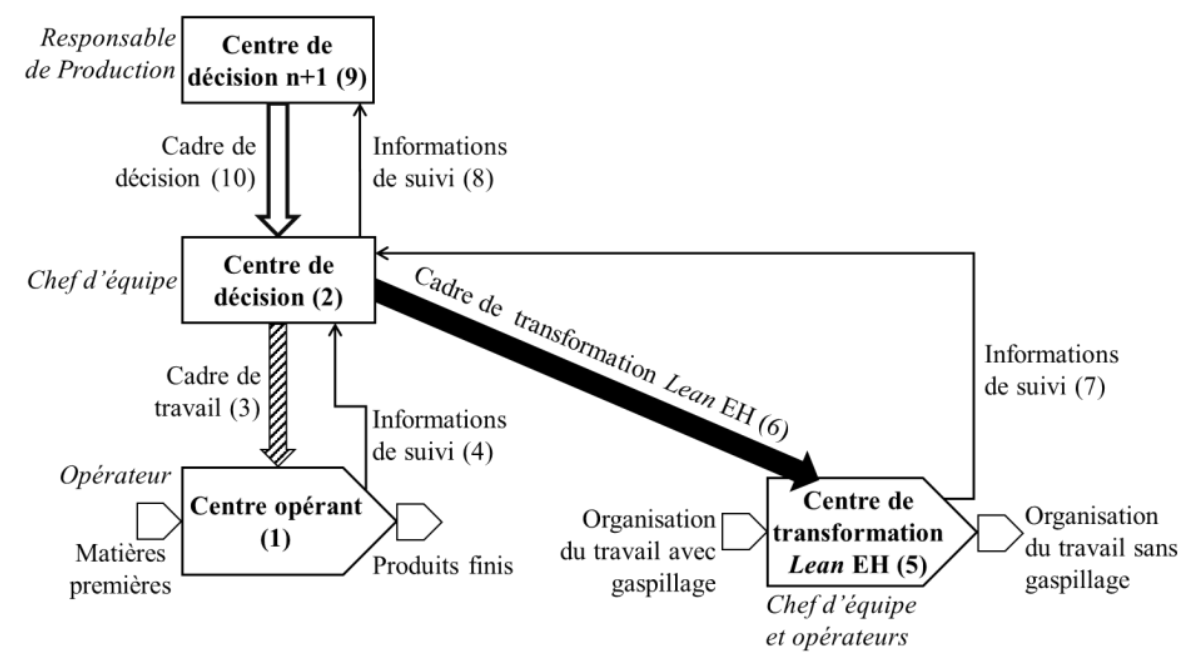

Figure 7. Application des modèle GRAI Lean EH à une entreprise Lean 
Il fixe comme variable de décision un choix plus large de moyens d'action qui ne focalise pas sur le respect strict des orientations du plan Hoshin Kanri de l'entreprise. Par exemple, il assouplit le principe du «zéro stock » pour laisser la possibilité au chef d'équipe de moduler la livraison au compte-goutte au regard de certains composants présentant des défauts récurrents.

Le centre de décision (2) adapte alors son cadre de travail au regard de ces nouveaux éléments issu du cadre de décision (10).

\section{Conclusion}

Nous avons proposé un modèle de performance qui cherche à pallier les limites rencontrées par les entreprises qui adoptent tout ou partie de la démarche Lean. Ce modèle repose sur une vision « acteur » des opérateurs de production qui les considère non pas comme des simples exécutants, mais comme des individus capables de déjouer les contraintes liées aux variabilités rencontrées au cours de la production. Cette vision « acteur » implique une approche de la performance qui non seulement prend en compte l'efficience opérationnelle, telle que la pratiquent les entreprises Lean, mais aussi l'efficience humaine $(\mathrm{EH})$. Nous avons nommé ce modèle « le modèle Lean $\mathrm{EH}$ ». Prendre en compte l'efficience humaine implique un autre regard sur les résultats et les moyens à prendre en compte pour évaluer l'efficience. Cette approche de la performance implique des interactions entre les managers et les opérateurs. Ces interactions portent essentiellement sur les marges de manœuvre qu'il est nécessaire de donner aux opérateurs afin qu'ils créent et déploient des modes opératoires pertinents pour la performance de l'entreprise en contexte Lean. La nature de ces interactions requiert un cadre de modélisation adapté.

Aussi, nous avons proposé un cadre de modélisation, basé sur la méthode GRAI et conjointement sur la méthodologie GRAI R\&D, adapté au contexte Lean avec cette vision « acteur ». Nous avons nommé les modèles proposés « modèles GRAI Lean $\mathrm{EH}$ » et nous avons proposé une illustration de leur applicabilité à travers un cas d'étude. Nos travaux de recherche présentent plusieurs limites qui ouvrent des perspectives pour l'avenir. Principalement, il nous faut valider de manière expérimentale les effets de notre modèle de performance Lean $\mathrm{EH}$ dans une entreprise Lean sur le long terme (au-delà d'un an). Ceci nécessite de nouveaux terrains d'expérimentation qui s'étaleront sur une période de plusieurs années afin de mesurer pleinement les effets de notre modèle. Préalablement, il nous faut opérationnaliser une méthodologie d'accompagnement qui permette aux membres d'une entreprise de faire évoluer leurs représentations pour passer de la vision « exécutante » à la vision « acteur » du modèle de performance Lean EH.

\section{Remerciements}

Nous remercions l'ensemble des responsables dans les 10 entreprises qui ont accepté de participer à notre enquête et à notre cas d'étude, et qui nous ont ainsi permis d'alimenter notre problématique scientifique, notre réflexion et nos propositions.

\section{Bibliographie}

[BAD 14] Badets, P., Pilniere, V., Merlo, C., (2014) A regulation mechanism based on work activity to improve Lean approach. Springer. Advances in Production Management Systems. Innovative and Knowledge-Based Production Management in a Global-Local World, Ajaccio, France. Vol.440.

[BES 93] Bescos, P.L., Dobler, P., Mendoza, C., Naulleau, G., (1993) Contrôle de gestion et management (2e éd.). Paris: Éditions Montchrestien.

[BHA 08] Bhasin, S. (2008). Lean and performance measurement. Journal of Manufacturing Technology Management, 19(5), 670-684. 
[BOU 10] Bourgeois, F., \& Gonon, O. (2010). Le Lean et l'activité humaine. Quel positionnement de l'ergonomie, convoquée par cette nouvelle doctrine de l'efficacité ? Activités, 1(7), 136-142.

[BOU 93] Bourguignon, A., (1993) Le modèle japonais de gestion. Paris: La Découverte.

[BYR 14] Byrne, A. (2014). Le virage Lean : Appliquez les principes du Lean à votre entreprise. Montreuil: Pearson.

[CAR 08] Caroly, S., Coutarel, F., Escriva, E., Roquelaure, Y., Schweitzer, J.-M., Daniellou, F., (2008) La prévention durable des TMS. Quels freins? Quels leviers d'action?. Paris: Direction Générale du Travail.

[CAS 09] Casey, D., (2009) The Role of Change Leadership in a Operations Excellence Transformation Model (Lulu Eds).

[DOU 84] Doumeingts, G., (1984) Méthode GRAI : méthode de conception des systèmes en productique (Thèse d'état). Université de Bordeaux.

[DRE 04] Drew, J., McCallum, B., Roggenhofer, S., (2004) Objectif lean. Réussir l'entreprise au plus juste: enjeux techniques et culturels. Paris: Eyrolles. Éditions d'Organisation.

[DUP 98] Dupont, L., (1998) La gestion industrielle. Paris: Hermès Science Publications.

[GIA 03] Giard, V., (2003) Gestion de la production et des flux (3e éd.). Paris: Economica.

[GIR 99] Girard, P., (1999) Etude de la conduite de la conception des produits manufacturés. Contribution à l'ingénierie des systèmes de conception. Université de Bordeaux.

[GIR 08] Giraud, F., Saulpic, O., Bonnier, C., Fourcade, F., Moisset, A., (2008) Contrôle de Gestion et Pilotage de la Performance (3e édition). Paris: Gualino Editeur.

[GUE 01] Guérin, F., Laville, A., Daniellou, F., Duraffourg, J., Kerguelen, A., (2001) Comprendre le travail pour le transformer: La pratique de l'ergonomie. Lyon: Anact.

[HOL 07] Holweg, M., (2007) The genealogy of lean production. Journal of Operations Management, 25(2), 420-437.

[JAG 14] Jagdish Rajaram J., Mantha, S. S., \& Rane, S. B. (2014). Development of framework for sustainable Lean implementation: an ISM approach. Journal of Industrial Engineering International, 10(3).

[LIK 04] Liker, J., (2004) The Toyota way : 14 management principles from the world's greatest manufacturer. New York: McGraw-Hill Professional.

[LIK 11] Liker, J., Franz, J. K., (2011) The Toyota way to continuous improvement : linking strategy and operational excellence to achieve superior performance. New York: McGraw-Hill Professional.

[MER 14] Merlo, C., Vicien, G., Ducq, Y., (2014) Interoperability Modelling Methodology for Product Design Organisations. Int. Journal of Production Research, Taylor \& Francis, Vol.52, Issue 15, pp. 100-120.

[OHN 88] Ohno, T., (1988) Toyota Production System. Beyond Large-Scale Production. New York: CRC Press.

[PAR 09] Pardi, T. (2009). Travailler chez Toyota : de l'emploi à vie à la course à la survie. La Revue de l'Ires, 62(3), 39.

[PIL 07] Pilnière, V., (2007) La gestion des risques professionnels : l'enjeu de l'accompagnement (Thèse de doctorat). Université de Pau et des Pays de l'Adour.

[UGH 12] Ughetto, P. (2012). Le lean : pensée et impensé d'une activité sans relâchement. Activités, 9(2), 148-167.

[WOM 12] Womack, J., Jones, D., (2012) Système Lean : penser l'entreprise au plus juste. Paris: Pearson France.

[WOM 93] Womack, J., Jones, D., Roos, D., (1993) Le système qui va changer le monde. Paris : Dunod. 